\section{Low-voltage small-size double-arm MEMS actuator}

\section{N. Biyikli, Y. Damgaci and B.A. Cetiner}

The fabrication and characterisation of a double-arm cantilever-type metallic DC-contact MEMS actuator with low pull-down voltage are reported. Bi-layer TiW cantilevers with an internal stress gradient were fabricated using a microwave-compatible fabrication process. Owing to its small size, cantilever length $(L=5-50 \mu \mathrm{m})$ and width $(W=2-40 \mu \mathrm{m})$, i.e. $\sim 10-100$ times smaller in lateral dimensions than a standard MEMS actuator, this actuator showed actuation voltages lower than $10 \mathrm{~V}$. RF measurements of the $10 \mu \mathrm{m}$-wide actuators yielded an average insertion loss less than $1 \mathrm{~dB}$ and isolation higher than $40 \mathrm{~dB}$ up to $25 \mathrm{GHz}$. The developed actuator is well suited for integration in reconfigurable microwave circuits and systems such as reconfigurable antennas and arrays.

Introduction: MEMS-based switches/actuators with low loss, high linearity and low DC-power consumption properties have proven to be well suited for various microwave applications such as reconfigurable cognitive wireless communication systems, adaptive matching networks, etc. [1-3]. However, the long term reliability of MEMS actuators, in particular during hot switching events, has been a major concern. The two primary failure mechanisms for DC-contact MEMS actuators are stiction (stuck closed) and increased contact resistance [4]. In addition to reliability issues, high actuation voltages (30$80 \mathrm{~V})$ and low switching speeds $(10-20 \mu \mathrm{s})$ may not be appropriate for next generation cognitive wireless communication applications. In these systems, the reconfigurability must be fast enough $(\sim$ less than $1 \mu \mathrm{s})$ in order to respond to short-term channel statistics and to support most dynamic adaptation schemes such as opportunistic beamforming schemes [5].

A high-reliability miniature RF-MEMS switched capacitor, similar to the standard capacitive membrane-type switch, has recently been reported [6]. The reported device with typical lateral dimensions of beamwidth $=10 \mu \mathrm{m}$ and beam length $=20 \mu \mathrm{m}$, achieves a pull-down voltage of $27 \mathrm{~V}$. In this Letter, we report a double-arm DC-contact small-size MEMS switch, where each cantilever length and width is as small as 10 and $2 \mu \mathrm{m}$, respectively. Fig. 1 shows the schematic and SEM picture of a typical actuator along with typical dimensions. The measured actuation voltages were consistently less than $10 \mathrm{~V}$. DCcontact MEMS actuators were chosen over their capacitive contact counterparts owing to their wide frequency range of operation. Also, a cantilever structure is used as opposed to a beam-membrane structure in order to precisely control the internal stress gradient, which in turn provides control on actuation voltage and isolation performance. It is also worth noting that the small size of MEMS actuators combined with cantilever design results in excellent mechanical properties such as increased spring constant and restoring force per contact area, which makes them less sensitive to residual stress or temperature variations compared to standard MEMS devices [6]. These are critically important features for reliable packaging processes.

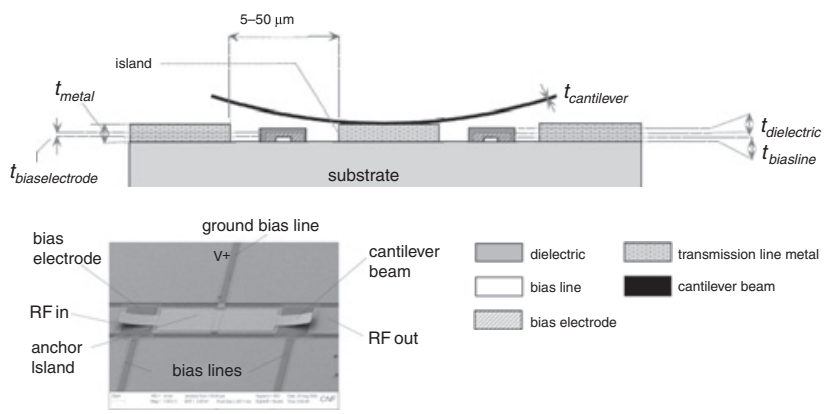

Fig. 1 Schematic and SEM picture of DC-contact double-arm cantilevertype MEMS actuator

$a$ Schematic

$b$ SEM picture of DC-contact double-arm cantilever-type MEMS actuator

Dimensions: cantilever width $=2-40 \mu \mathrm{m}, t_{\text {metal }}=1.0 \mu \mathrm{m}, t_{\text {cantilever }}=0.2$

$0.4 \mu \mathrm{m}, t_{\text {biaselectrode }} \sim 0.2 \mu \mathrm{m}, t_{\text {biasline }}=0.1-0.2 \mu \mathrm{m}, t_{\text {dielectric }} \sim 0.2 \mu \mathrm{m}$

Design and microfabrication: The double-arm MEMS actuators were realised on a microwave coplanar waveguide (CPW) transmission line and fabricated on a synthetic quartz substrate using a six-layer microwave-compatible fabrication process. The relative dielectric constant and loss tangent of the quartz substrate for the frequency range $0.1-30 \mathrm{GHz}$ are 3.9 and $\sim 0.0002$, respectively. Fig. 2 shows the step-by-step fabrication procedure. First, the bias lines were formed by a $\sim 150 \mathrm{~nm}$-thick $\mathrm{Ti} / \mathrm{Cr}$ layer deposited by DC-sputtering. Bias electrode pads were formed with an e-beam evaporated $\sim 200 \mathrm{~nm}$ thick $\mathrm{Ti} / \mathrm{Cu}$ layer. Afterwards, bias lines and bias electrodes were passivated with a $250 \mathrm{~nm}$-thick $\mathrm{SiO}_{2}$ dielectric layer deposited at $275^{\circ} \mathrm{C}$ with a plasma-enhanced chemical vapour-deposition (PECVD) system. In the fourth step, using the e-beam evaporation system, a $1.0 \mu \mathrm{m}$-thick $\mathrm{Ti} / \mathrm{Cu}$ metal layer was deposited as a microwave $\mathrm{CPW}$ transmission line. A thick $(\sim 2 \mu \mathrm{m})$ amorphous $\mathrm{Si}$ (a:Si) sacrificial layer was deposited in the PECVD system at $200^{\circ} \mathrm{C}$. Our process optimisation efforts showed that the planarisation of the sacrificial layer was of critical importance for the successful release and mechanical integrity of subsequently deposited cantilevers. A chemical-mechanical polishing (CMP) technique was used to planarise the a:Si sacrificial layer. The polishing process was stopped when $\sim 0.1 \mu \mathrm{m}$ a:Si layer was left on top of $\mathrm{Ti} / \mathrm{Cu} \mathrm{CPW}$. In the final step, a $\sim 0.4 \mu \mathrm{m}$-thick TiW layer was DC-sputtered using $25 \mathrm{sccm}$ Ar and a TiW (90/10\% wt) target. The TiW cantilevers were patterned using a $\mathrm{CF}_{4}$-based reactive ion etch (RIE). The release of the cantilevers was completed in a $\mathrm{XeF}_{2}$-etcher system where the underlying a:Si sacrificial layer was effectively etched at lateral etch rates more than $10 \mu \mathrm{m} / \mathrm{min}$.
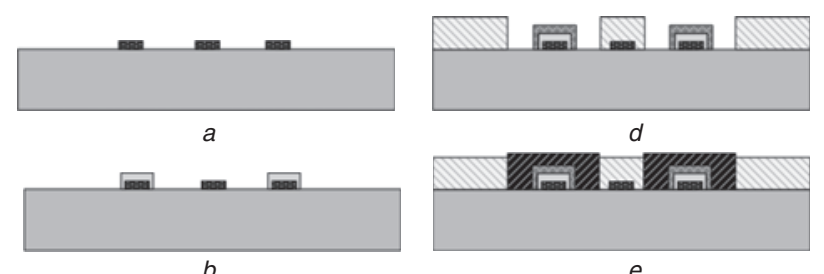

$b$
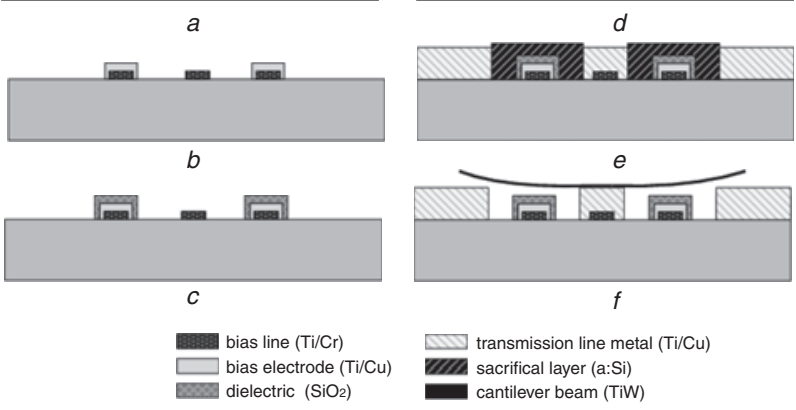

Fig. 2 Fabrication process steps for double-arm DC-contact MEMS actuators

$a$ Deposition of $\mathrm{Ti} / \mathrm{Cr} \mathrm{DC}$ bias lines

$b \mathrm{Ti} / \mathrm{Cu}$ bias electrode metal deposition

$c \mathrm{SiO}_{2}$ dielectric layer deposition

$d \mathrm{Ti} / \mathrm{Cu}$ transmission line (CPW) metal formation

$e$ a:Si sacrificial layer deposition and planarisation

$f$ TiW cantilever formation and release process

The cantilevers curled upwards as expected after the release process, owing to the internal stress gradient within the deposited TiW. The stress gradient was achieved by depositing the TiW under two different sputtering conditions: the bottom half was deposited under 16 mtorr chamber pressures, which resulted in a compressively stressed layer, whereas the top half was sputtered under 21 mtorr, resulting in a top layer with tensile stress. The corresponding internal stress gradient was about $1000 \mathrm{MP}$. By increasing/decreasing the pressure difference of the two TiW layers, it is possible to tune the internal stress gradient to achieve the desired amount of cantilever bending.

Results: MEMS actuators of various lateral cantilever sizes with length $(L=5-50 \mu \mathrm{m})$ and width $(W=2-40 \mu \mathrm{m})$ were fabricated. Fig. 3 shows pictures of different devices taken by a scanning electron microscope (SEM). The amount of curling increases with the length of cantilever. The actuation (pull-down) voltage of devices was measured on-wafer using a probe station and a DC bias source. All MEMS actuators exhibited actuation voltages below $20 \mathrm{~V}$. For cantilever widths smaller than $20 \mu \mathrm{m}$, actuation voltages lower than $10 \mathrm{~V}$ were consistently measured. 

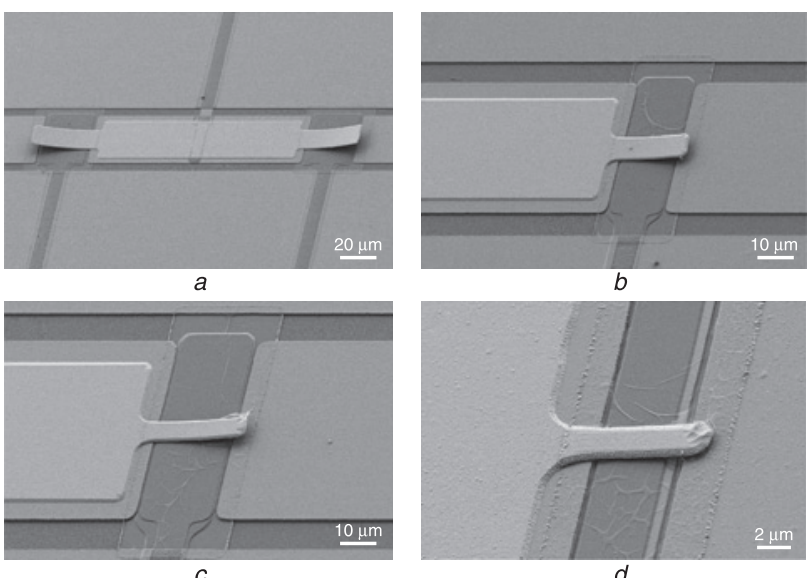

$d$

Fig. 3 SEM pictures of DC-contact RF MEMS double-arm actuators

$a L=50 \mu \mathrm{m}, W=20 \mu \mathrm{m}$ (both arms shown)

$b L=25 \mu \mathrm{m}, W=10 \mu \mathrm{m}$

$c L=20 \mu \mathrm{m}, W=5 \mu \mathrm{m}$

$d L=10 \mu \mathrm{m}, W=2 \mu \mathrm{m}$

The RF performance of fabricated MEMS actuators has been characterised by measuring small-signal $S$-parameters using a probe station with $40 \mathrm{GHz}$ RF probes and a $26 \mathrm{GHz}$ network analyser. The actuators exhibited excellent isolation (an average of $50 \mathrm{~dB}$ ) of the zero-bias open-state (OFF-state) for any of the cantilever dimensions as shown in Fig. $4 a$. Fig. $4 b$ shows the measured insertion losses of the biased actuator (ONstate) for different cantilever dimensions as a function of frequency. As can be seen in Fig. 4b, insertion loss steadily increases for decreasing membrane dimensions, which is an indication that the contact resistance gets larger as the membrane width gets smaller. The insertion loss is relatively constant over the frequency range of $100 \mathrm{MHz}-25 \mathrm{GHz}$, which is consistent with the constant loss prediction of real resistance, $S_{21}=2 Z_{o} /\left(2 Z_{o}+\right.$ $R_{c}$ ), where $R_{c}$ is the contact resistance. The average insertion loss is at around $0.2 \mathrm{~dB}$ for $40 \mu \mathrm{m}$-wide actuators, while it degrades to around $2 \mathrm{~dB}$ for $2 \mu \mathrm{m}$-wide actuators.
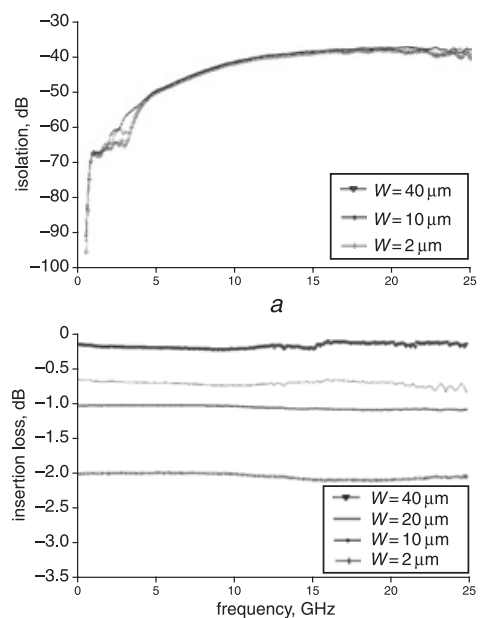

$b$

Fig. 4 Measured isolation and insertion loss of fabricated DC-contact MEMS double-arm actuator for membrane widths, $W=40,20,10$, and $2 \mu m$
Conclusions: Significant progress towards obtaining highly reliable small size MEMS actuators with low actuation voltage and fast switching speed is reported. We have fabricated and characterised the DC and RF performance of small-size double-arm DC-contact MEMS actuators of cantilever type with an internal stress gradient. The actuators showed promising performance results. To improve the insertion loss performance of the actuators with a small cantilever width, we are currently optimising mechanical switch design using novel contact architectures and investigating various alloy electric contact metallurgies with desired electrical and mechanical properties. We are also developing new nanofabrication processes as a way of reducing contact resistance and improving reliability.

Acknowledgments: This work was supported in part by the National Institute of Justice under grant 2007-IJ-CX-K025. This work was performed in part at the Cornell NanoScale Facility, a member of the National Nanotechnology Infrastructure Network, which is supported by the National Science Foundation (grant ECS-0335765).

(C) The Institution of Engineering and Technology 2009

21 January 2009

doi: 10.1049/el.2009.0207

Y. Damgaci and B.A. Cetiner (Department of Electrical and Computer Engineering, Utah State University, Logan, UT 84341, USA)

N. Biyikli (Bilkent University, UNAM-Institute of Materials Science and Nanotechnology, 06800 Bilkent, Ankara, Turkey)

E-mail: biyikli@unam.bilkent.edu.tr

\section{References}

1 Rebeiz, G.M.: 'RF MEMS theory, design and technology' (Wiley, New York, 2003)

2 Duffy, S., Bozler, C., Rabe, S., Knecht, J., Travis, L., Wyatt, P., Keast, C., and Gouker, M.: 'MEMS microswitches for reconfigurable microwave circuitry', IEEE Microw. Wirel. Compon. Lett., 2001, 11, (3), pp. 106-108

3 Cetiner, B.A.: 'MIMO antenna technology for wireless communications', (Electrical Engineering and Applied Signal Processing Series, CRC Press, 2006)

4 Dickrell, D.J., and Dugger, M.T.: 'Electrical contact resistance degradation of a hot-switched simulated metal MEMS contact', IEEE Trans. Compon. Packag. Technol., 2007, 30, (1), pp. 75-80

5 Viswanath, P., Tse, D., and Laroia, R.: 'Opportunistic beamforming using dumb antennas', IEEE Trans. Inf. Theory, 2002, 48, (5), pp. 1277-1294

6 Lakshminarayanan, B., Mercier, D., and Rebeiz, G.M.: 'High-reliability miniature RF-MEMS switched capacitors,', IEEE Trans. Microw. Theory Tech., 2008, 56, (4), pp. 971-981 